\title{
Heart failure with preserved ejection fraction as an independent risk factor of mortality after cardiothoracic surgery
}

Lee S. Nguyen, MD, ${ }^{\mathrm{a}, \mathrm{b}}$ Pierre Baudinaud, MD, ${ }^{\mathrm{a}}$ Alain Brusset, $\mathrm{MD},{ }^{\mathrm{a}}$ Florence Nicot, MD, ${ }^{\mathrm{a}}$

Louis Pechmajou, MD, ${ }^{b}$ Joe-Elie Salem, $\mathrm{MD}, \mathrm{PhD},{ }^{\mathrm{c}}$ Philippe Estagnasie, $\mathrm{MD},{ }^{\mathrm{a}}$ and Pierre Squara, $\mathrm{MD}^{\mathrm{a}}$

\section{ABSTRACT}

Background: The prognostic role of heart failure with preserved ejection fraction (HFpEF) remains unclear. This study aimed to assess HFpEF prognostic value after cardiothoracic surgery, adjusting for European System for Cardiac Operative Risk (EuroSCORE II) criteria.

Methods: Patients with left ventricular ejection fraction (LVEF) $\geq 50 \%$ undergoing cardiothoracic surgery between 2012 and 2016 were included. Patients with HFpEF were compared to control patients with LVEF $\geq 50 \%$. HFpEF was defined following 2016 European Society of Cardiology guidelines: LVEF $\geq 50 \%$, symptomatic HF with New York Heart Association (NYHA) class 2 or greater, elevated brain natriuretic peptide (BNP) and relevant echocardiographic findings (LV hypertrophy, LA enlargement, or diastolic filling anomaly). The primary endpoint was in-hospital mortality, and the secondary endpoint was postoperative shock. Multivariate analyses were performed to determine mortality and shock risk-factors.

Results: Among 1743 patients, 427 (24.5\%) presented HFpEF. HFpEF was highly associated with in-hospital mortality (hazard ratio $=1.86 ; 95 \%$ confidence interval $[\mathrm{CI}], 1.16-2.98 ; P=.01)$. This association remained independent when adjusting for EuroSCORE II (adjusted hazard ratio $=1.6 ; 95 \%$ CI, 1.0-2.6; $P=.049)$. Postoperative shock occurred more in HFpEF than in control patients $(17.8 \%$ vs $6.7 \% ; P<.001)$. HFpEF was an independent risk factor of postoperative shock (adjusted odds ratio $=2.9 ; 95 \% \mathrm{CI}, 1.5-3.0 ; P<.001$ ).

Conclusions: HFpEF was an independent risk-factor of mortality and postoperative shock after cardiothoracic surgery, after adjustment regarding EuroSCORE II. (J Thorac Cardiovasc Surg 2018;156:188-93)

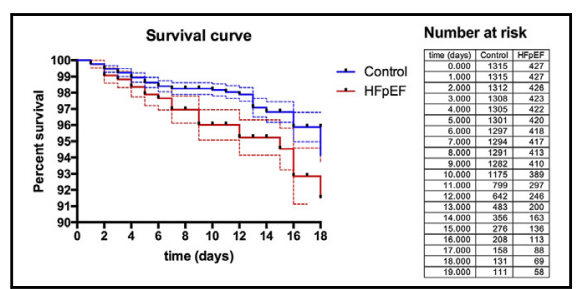

Survival curve representing incidence of postoperative in-hospital mortality.

\section{Central Message}

Heart failure with preserved ejection fraction is an independent risk factor of adverse events including death after cardiothoracic surgery. These results remain true after adjusting for EuroSCORE II.

\section{Perspective}

Heart failure with preserved ejection fraction (HFpEF) was redefined with the more strict 2016 ESC guidelines. The prognostic impact of HFPEF on cardiothoracic surgery has not been evaluated in a global cardiac surgery cohort. Results from the RIPOSTE prospective database show that HFPEF is indeed a risk factor of mortality and question the need to systematically look for HFPEF before surgery.

See Editorial Commentary page 194.
Heart failure $(\mathrm{HF})$ with preserved ejection fraction $(\mathrm{HFpEF})$ is a public health issue growing worldwide. Its prevalence is $50 \%$ of patients aged 65 years or older. ${ }^{1}$ Although heart

From the ${ }^{\mathrm{a} C M C}$ Ambroise Paré, Critical Care Medicine Department, Neuilly-surSeine, France; ${ }^{\mathrm{b}} \mathrm{AP}-\mathrm{HP}$, Pitié-Salpêtrière Hospital, Clinical Investigation Center-Paris-Est, Paris, France; and ${ }^{\mathrm{c}} \mathrm{AP}-\mathrm{HP}$, Mondor Hospital, Cardiology Department, Créteil, France.

Drs Nguyen and Baudinaud contributed equally to this work.

Received for publication July 18, 2017; revisions received Jan 21, 2018; accepted for publication Feb 2, 2018; available ahead of print March 9, 2018.

Address for reprints: Lee S. Nguyen, MD, Critical Care Medicine Department, CMC Ambroise Paré, 25 Boulevard Victor Hugo, 92200 Neuilly-sur-Seine, France (E-mail: nguyen.lee@icloud.com).

$0022-5223 / \$ 36.00$

Copyright (c) 2018 by The American Association for Thoracic Surgery

https://doi.org/10.1016/j.jtcvs.2018.02.011 failure with reduced ejection fraction (HFrEF) is associated with increased mortality after cardiac surgery, the prognostic impact of $\mathrm{HFpEF}$ remains unclear. ${ }^{2}$ Indeed, studies concluded with conflicting results in cardiac surgery, ${ }^{3,4}$ whereas HFpEF was identified as an important prognostic factor in other settings.

The European System for Cardiac Operative Risk (EuroSCORE II) is the most widely used risk stratification

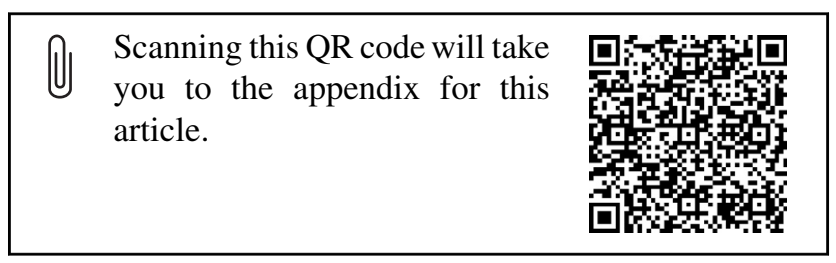




$\begin{array}{ll}\text { Abbreviations and Acronyms } \\ \text { AUROC } & \text { receiver operating characteristics } \\ & \text { area under the curve } \\ \text { BNP } & =\text { brain natriuretic peptide } \\ \text { CABG } & \text { coronary artery bypass graft } \\ \text { ESC } & =\text { European Society of Cardiology } \\ \text { EuroSCORE II = } & \text { European System for Cardiac } \\ & \text { Operative Risk } \\ \text { HF } & =\text { heart failure } \\ \text { HFpEF } & =\text { heart failure with preserved } \\ & \text { ejection fraction } \\ \text { HFrEF } & =\text { heart failure with reduced ejection } \\ & \text { fraction } \\ \text { HR } & =\text { hazard ratio } \\ \text { LVEF } & =\text { left ventricular ejection fraction } \\ \text { NYHA } & =\text { New York Heart Association } \\ \text { PA } & =\text { pulmonary artery }\end{array}$

score in cardiothoracic surgery. ${ }^{8}$ It is a multivariable score allowing the calculation of risk of death before undertaking cardiac surgery, and it can help physicians and patients with the advisability of an operation with an assessment of the risk/benefit ratio. Although it accounts for numerous variables, EuroSCORE II was released in 2012 and did not include HFpEF, as later defined. ${ }^{9}$

This study aimed to assess HFpEF prognostic impact regarding postoperative outcomes after cardiothoracic surgery; comparing patients with $\mathrm{HFpEF}$ and control patients with left ventricular ejection fraction (LVEF) $>50 \%$. Analyses were adjusted on EuroSCORE II; other confounding factors included age, elevated systolic pulmonary artery (PA) pressure, impaired renal function, emergency procedure, and type of procedure (association with mitral valve or thoracic aorta).

\section{METHODS \\ Study Population}

From the January 1, 2012, to September 30, 2016, all adult patients undergoing cardiac surgery with cardiopulmonary bypass were screened; they were included if their LVEF was $50 \%$ or greater. Exclusion criteria were: age less than 18 years and reinterventions during the same hospitalization.

\section{Definitions and Data Collection}

HFpEF status was evaluated preoperatively. It was defined per the 2016 European Society of Cardiology (ESC) guidelines for acute and chronic HF: symptomatic HF (at least NYHA class 2) associated with LVEF $\geq 50 \%$ and elevated levels of natriuretic peptides with at least one of the additional criteria: relevant structural heart disease (left ventricular hypertrophy, left atrium enlargement) or diastolic dysfunction (elevated filling pressures assessed by E/Ea wave ratio). ${ }^{9}$

LVEF and left atrium volume index were measured by 2-dimensional transthoracic echocardiography using the Simpson method. E/Ea wave ratio was measured using the Doppler method. Left ventricular mass index was computed using Devereux formula from 2-dimensional transthoracic echocardiography. ${ }^{10} \mathrm{BNP}$ was considered elevated when a superior to normal laboratory value ( $\geq 100 \mathrm{ng} / \mathrm{L}$ ) was measured.

EuroSCORE II was computed as described in the original article. ${ }^{8}$ It included age, sex, NYHA functional class, angina symptoms, insulin-dependent diabetes mellitus, extracardiac arteriopathy, chronic pulmonary dysfunction, neurologic or musculoskeletal dysfunction severely affecting mobility, previous cardiac surgery, renal function with creatinine clearance, active endocarditis, critical preoperative state, LVEF, recent myocardial infarction, PA systolic pressure, procedure urgency, and weight of the procedure (coronary artery bypass graft, valve surgery, or thoracic aorta). All variables used were defined as in the original EuroSCORE II. There were no missing data. Throughout the study, all surgery procedures were performed by the same team of surgeons, all of whom performed the same proportion of procedures.

Data come from the Registry for the Improvement of Postoperative Outcomes in Cardiac and Thoracic Surgery database, which is registered at clinicaltrials.gov under NCT03209674. Data were collected prospectively for each patient: demographic data, HFpEF status, variables required for the computation of EuroSCORE II, use of catecholamines after surgery, and in-hospital mortality. Echocardiographic parameters were prospectively collected in the database. Data were anonymized per national regulations and used with the approval of an institutional review board committee and data collection was authorized under French national legislation (CNIL, registration number 2029657; AMR003).

\section{Clinical Outcomes}

Main endpoint was in-hospital mortality. It was defined similarly to the EuroSCORE II study: death occurring in the same hospital where the operation took place before discharge from the hospital.

Secondary endpoint was the postoperative need for inotropic support in the first 24 hours after surgery. Specifically, it was defined as the use of catecholamines (dobutamine, norepinephrine and/or epinephrine) to achieve standard therapeutic targets in cardiac surgery intensive care unit: cardiac index $>2.2 \mathrm{~L} / \mathrm{min} / \mathrm{m}^{2}$ assessed with echocardiography or Swan-Ganz catheter when available, mean blood pressure $>65 \mathrm{~mm} \mathrm{Hg}$, and urine output $>30 \mathrm{~mL} /$ hour. Adequate volume resuscitation was also performed.

\section{Statistical Analysis}

Descriptive analyses were conducted and expressed, for continuous variables as mean \pm standard deviation and for categorical variables as number of occurrences (percentage). Intergroup comparisons were assessed using parametric $t$ tests. Distribution was assessed using Kolmogov-Smirnov test. Nonparametric tests were performed when appropriate. Continuous variables which were not normally distributed were expressed as median (interquartile range). Discrimination performance of EuroSCORE II toward in-hospital mortality and shock was assessed using receiver-operator characteristics area under the curve (AUROC).

Association between mortality (time to death), EuroSCORE II, and HFpEF were assessed using Cox regression analyses with a forward likelihood-ratio method. A multivariable model including the 7 variables with mortality most associated (age, mitral valve surgery, thoracic aorta associated-procedure, emergency procedure, severe pulmonary artery hypertension $>50 \mathrm{~mm} \mathrm{Hg}$, eGFR $<50 \mathrm{~mL} / \mathrm{min}$ and $\mathrm{HFpEF}$ ) was also computed as a sensitivity analysis.

Association between postoperative need for inotropic support, EuroSCORE II, and HFpEF was assessed using binary logistic regression analyses. Logistic regression was used in the latter analysis because postoperative need for inotropic support was defined as immediate use of catecholamines after surgery; hence, survival-type analysis such as Cox would not be appropriate. A multivariable model including 8 confounding variables associated with the need for inotropic support (age, isolated coronary artery bypass grafting [CABG], emergency procedure, severe 
TABLE 1. Baseline characteristics

\begin{tabular}{|c|c|c|c|}
\hline Characteristics & $\begin{array}{c}\text { Control } \\
(\mathbf{n}=1316)\end{array}$ & $\begin{array}{c}\text { HFpEF } \\
(n=427)\end{array}$ & $\begin{array}{c}P \\
\text { value }\end{array}$ \\
\hline Age (y) & $67.3 \pm 11.3$ & $71.5 \pm 10.8$ & $<.001$ \\
\hline Male sex & $974(74.0)$ & $263(61.0)$ & $<.001$ \\
\hline Height $(\mathrm{cm})$ & $170 \pm 8.9$ & $168 \pm 9.4$ & $<.001$ \\
\hline Weight (kg) & $77.6 \pm 15$ & $74.3 \pm 15.6$ & $<.001$ \\
\hline Body mass index $\left(\mathrm{kg} / \mathrm{m}^{2}\right)$ & $26.9 \pm 4.6$ & $26.4 \pm 5$ & .54 \\
\hline EuroSCORE II (\%) & $1.4(0.9 ; 2.6)$ & $2.9(1.6 ; 4.6)$ & $<.001$ \\
\hline $\begin{array}{l}\text { Mildly reduced kidney } \\
\text { function }\end{array}$ & $636(48.3)$ & $189(44.3)$ & .144 \\
\hline $\begin{array}{l}\text { Severely reduced kidney } \\
\text { function }\end{array}$ & 273 (20.7) & $157(36.8)$ & $<.001$ \\
\hline Hemodialysis & $13(1.0)$ & $4(0.9)$ & .926 \\
\hline Severe pulmonary hypertension & $25(1.9)$ & $65(15.2)$ & $<.001$ \\
\hline Mild pulmonary hypertension & $418(31.8)$ & $205(48.0)$ & $<.001$ \\
\hline Poor mobility & $11(0.8)$ & $11(2.6)$ & .005 \\
\hline Diabetes mellitus on insulin & $75(5.7)$ & $19(4.4)$ & .321 \\
\hline Extracardiac arteriopathy & $164(12.5)$ & $34(8.0)$ & .011 \\
\hline Recent myocardial infarction & $63(4.8)$ & $10(2.3)$ & .028 \\
\hline Class 4 angina & $24(1.8)$ & $3(0.7)$ & .103 \\
\hline Critical preoperative state & $16(1.2)$ & $10(2.3)$ & .095 \\
\hline Active endocarditis & $37(2.8)$ & $17(4.0)$ & .226 \\
\hline NYHA class 2 & $220(16.7)$ & 213 (49.9) & $<.001$ \\
\hline NYHA class 3 & $142(10.8)$ & $186(43.6)$ & $<.001$ \\
\hline NYHA class 4 & $13(1.0)$ & $28(6.6)$ & $<.001$ \\
\hline COPD & $66(5.0)$ & $42(9.8)$ & $<.001$ \\
\hline
\end{tabular}

Laboratory data

Creatininemia $(\mu \mathrm{mol} / \mathrm{L}) \quad 17.0(9.0 ; 87.0) 14.5(9.0 ; 93.0) \quad .566$

$\mathrm{BNP}(\mathrm{pg} / \mathrm{mL}) \quad 64(32 ; 142) \quad 266(159 ; 570)<.001$

\begin{tabular}{lccc} 
Echocardiographic parameters & & & \\
LVM index $\left(\mathrm{g} / \mathrm{m}^{2}\right)$ & $112.3 \pm 39.2$ & $124.2 \pm 36.7$ & $<.001$ \\
LAV index $\left(\mathrm{mL} / \mathrm{m}^{2}\right)$ & $25.0 \pm 15.9$ & $36.7 \pm 20.7$ & $<.001$ \\
E/Ea ratio & $8.1 \pm 5.0$ & $12.6 \pm 6.1$ & $<.001$ \\
LVEF $(\%)$ & $64.4 \pm 7.9$ & $64.5 \pm 8.8$ & .45 \\
Systolic PA pressure & $34.4 \pm 9.4$ & $42.1 \pm 15.0$ & $<.001$ \\
$\quad(\mathrm{~mm} \mathrm{Hg})$ & & & \\
Surgery procedure & & & \\
Isolated CABG & $719(54.6)$ & $66(15.5)$ & $<.001$ \\
CABG & $866(65.8)$ & $152(35.6)$ & $<.001$ \\
Valve procedure & $544(41.3)$ & $357(83.6)$ & $<.001$ \\
Aortic valve & $395(30.0)$ & $225(52.7)$ & $<.001$ \\
Mitral valve & $149(11.3)$ & $145(34.0)$ & $<.001$ \\
Mitral repair & $101(7.7)$ & $63(14.8)$ & $<.001$ \\
Mitral replacement & $48(3.6)$ & $82(19.2)$ & $<.001$ \\
Tricuspid valve & $27(2.1)$ & $49(11.5)$ & $<.001$ \\
Previous cardiac surgery & $48(3.6)$ & $31(7.3)$ & .002 \\
Surgery on thoracic aorta & $95(7.2)$ & $23(5.4)$ & .191 \\
Emergency & $56(4.3)$ & $15(3.5)$ & .5 \\
\hline
\end{tabular}

TABLE 1. Continued

\begin{tabular}{lccr}
\hline \multicolumn{1}{c}{ Characteristics } & $\begin{array}{c}\text { Control } \\
(\mathbf{n = 1 3 1 6 )}\end{array}$ & $\begin{array}{c}\text { HFpEF } \\
(\mathbf{n = 4 2 7 )}\end{array}$ & $\begin{array}{c}\boldsymbol{P} \\
\text { value }\end{array}$ \\
\hline Urgent surgery & $183(13.9)$ & $50(11.7)$ & .247 \\
Salvage surgery & $1(0.08)$ & $1(0.2)$ & .402 \\
Single non-CABG procedure & $366(27.8)$ & $191(44.7)$ & $<.001$ \\
2 procedures & $218(16.6)$ & $154(36.1)$ & $<.001$ \\
3 procedures or more & $22(1.7)$ & $18(4.2)$ & .002 \\
\hline
\end{tabular}

All definitions are these of the EuroSCORE II. Continuous data are presented as mean \pm standard deviation, categorical data as number (percentage). Non-normally distributed continuous data are presented as median (interquartile range). EuroSCORE, European System for Cardiac Operative Risk; NYHA, New York Heart Association dyspnea class; $C O P D$, chronic obstructive pulmonary disease; $B N P$, brain natriuretic peptide; $L V M$, left ventricular mass; $L A V$, left atrium volume; $L V E F$, left ventricular ejection fraction; $P A$, pulmonary artery; $C A B G$, coronary artery bypass graft.

pulmonary artery hypertension $>50 \mathrm{~mm} \mathrm{Hg}$, eGFR $<50 \mathrm{~mL} / \mathrm{min}, \mathrm{HFpEF}$, 3 or more combined procedures, and $\mathrm{BNP}$ ) were also computed as a sensitivity analysis.

The minimum number of patients to achieve a power of $1-\beta=0.8$ and a primary risk $\alpha=0.05$ was estimated to be 1514 assuming incidence of postoperative mortality between $4 \%$ and $5 \%$ and, to be $83 \%$ higher in HFpEF patients than control group. ${ }^{11}$

All calculations were performed with SPSS version 23.0 (IBM, Armonk, NY). Figures were drawn using GraphPad Prism version 7.0 (GraphPad Software, La Jolla Calif).

\section{RESULTS \\ Baseline Characteristics}

The study included 1743 patients with LVEF $\geq 50 \%$. Among them, 427 (24.5\%) fulfilled criteria for HFpEF. Baseline characteristics, including echocardiographic parameters, are presented in Table 1. EuroSCORE II, accounting for all these characteristics in addition to the type of surgery, was higher in HFpEF patients than control patients $(4.5 \%$ vs $2.4 \% ; P<.001)$. Median follow-up time was 12 days. $^{10,12}$

\section{In-hospital Mortality}

Overall postoperative in-hospital mortality was $4.1 \%$. EuroSCORE II accurately discriminated in-hospital mortality with an AUROC of $0.81(P<.001$; Figure E1). Postoperative in-hospital mortality was higher in patients with HFpEF than in control patients $(7.0 \%$ vs $3.2 \%$; $P$ value $<.001)$.

Univariate survival analysis showed HFpEF was associated with in-hospital mortality (hazard ratio $[\mathrm{HR}]=1.86 ; 95 \%$ confidence interval $[\mathrm{CI}], 1.16-2.98$; $P=.01)$. Multivariate survival analysis adjusted on EuroSCORE II showed HFpEF remained independently associated with postoperative in-hospital mortality (adjusted HR $=1.6 ; 95 \%$ CI, 1.0-2.6; $P=.049$; Figure 1).

Multivariable model built with adjustment regarding confounding variables associated with in-hospital mortality found similar results with an adjusted $\mathrm{HR}$ of 1.73 $(1.004-2.977 ; P$ value $=.048$; Table E1 $)$. 


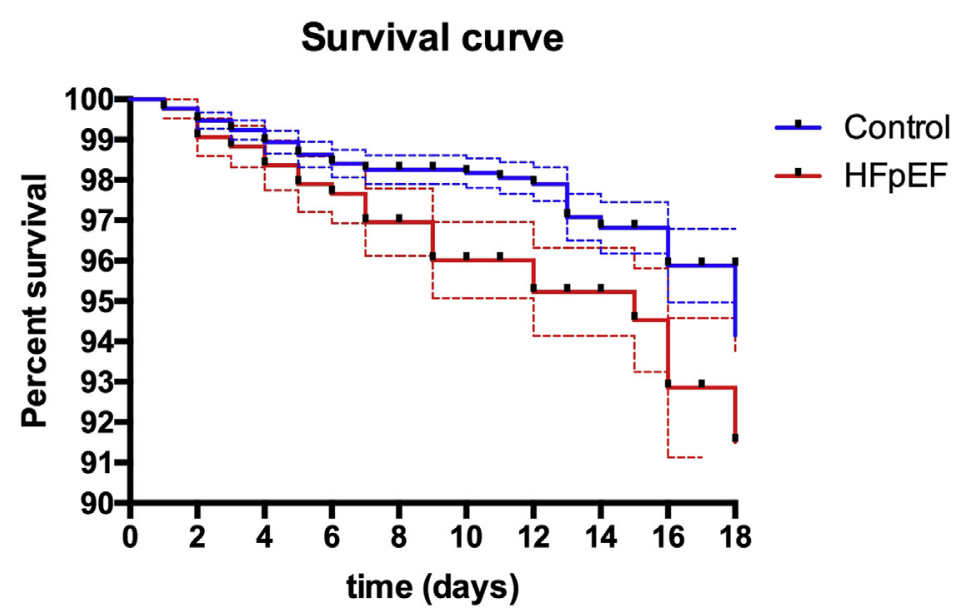

\section{Number at risk}

\begin{tabular}{|r|r|r|}
\hline time (days) & Control & HFpEF \\
\hline 0.000 & 1315 & 427 \\
\hline 1.000 & 1315 & 427 \\
\hline 2.000 & 1312 & 426 \\
\hline 3.000 & 1308 & 423 \\
\hline 4.000 & 1305 & 422 \\
\hline 5.000 & 1301 & 420 \\
\hline 6.000 & 1297 & 418 \\
\hline 7.000 & 1294 & 417 \\
\hline 8.000 & 1291 & 413 \\
\hline 9.000 & 1282 & 410 \\
\hline 10.000 & 1175 & 389 \\
\hline 11.000 & 799 & 297 \\
\hline 12.000 & 642 & 246 \\
\hline 13.000 & 483 & 200 \\
\hline 14.000 & 356 & 163 \\
\hline 15.000 & 276 & 136 \\
\hline 16.000 & 208 & 113 \\
\hline 17.000 & 158 & 88 \\
\hline 18.000 & 131 & 69 \\
\hline 19.000 & 111 & 58 \\
\hline
\end{tabular}

FIGURE 1. Survival curve representing incidence of postoperative in-hospital mortality. $H F p E F$, Heart failure with preserved ejection fraction.

\section{Postoperative Need for Inotropic Support}

Overall, 165 patients $(9.5 \%)$ presented a need for inotropic support in the first 24 hours. This condition was more frequent in HFpEF patients than in control patients $(17.8 \%$ vs $6.7 \% ; P<.001)$. EuroSCORE II accurately discriminated this need for inotropic support with an AUROC of $0.77(P<.001$; Figure E2).

Univariate logistic regression analysis showed that HFpEF was associated with a postoperative need for inotropic support (odds ratio $[\mathrm{OR}]=3.0 ; 95 \% \mathrm{CI}$,
2.15-4.15; $P<.001)$. Multivariate logistic regression analysis, with adjustment regarding EuroSCORE II, showed that HFpEF remained independently associated with postoperative need for inotropic support (adjusted $\mathrm{OR}=2.1 ; 95 \%$ CI, $1.45-2.97 ; P<.001)$.

A multivariable model built with adjustment regarding confounding variables associated with postoperative need for inotropic support found similar results with an adjusted HR of $1.52(95 \% \mathrm{CI}, 1.04-2.23 ; P<.001$; Table E2).

\section{Overall cohort}

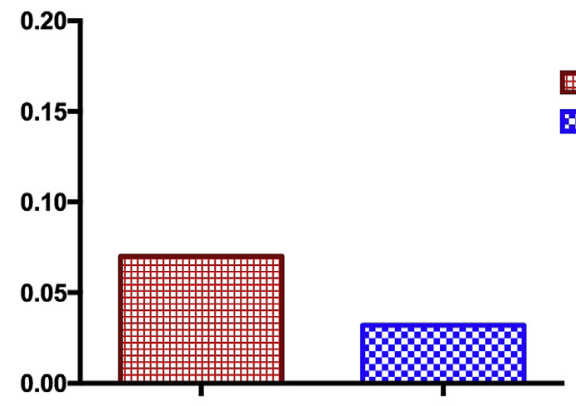

Aortic valve surgery

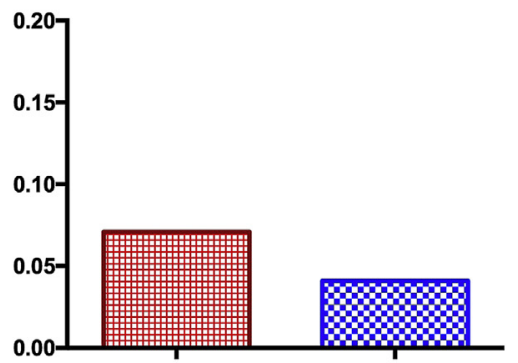

Isolated CABG

HFPEF

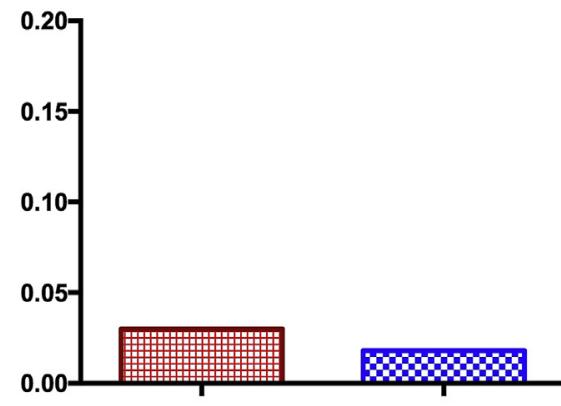

Mitral valve surgery

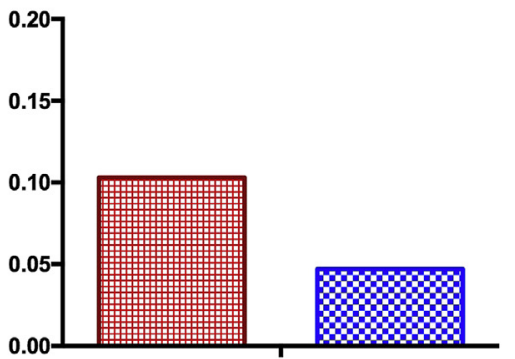

FIGURE 2. Incidence of postoperative mortality. Results are shown in patients with heart failure with preserved ejection fraction $(H F p E F)$ and control patients, in the overall cohort and in subgroup analyses (types of procedures). Intergroup comparisons were not applicable in subgroup analyses because of sample size. $C A B G$, Coronary artery bypass grafting. 


\section{Subgroup Analyses on Type of Surgery}

Although the sample size did not allow for sufficient power as prespecified in the statistical section, patients with HFpEF tended to have an increased incidence of in-hospital mortality than control patients did in isolated CABG surgery $(3.0 \%$ vs $1.8 \% ; P=.49)$, in aortic valve surgery $(7.1 \%$ vs $4.1 \% ; P=.09)$, and in mitral valve surgery $(10.3 \%$ vs $4.7 \% ; P=.06$; Figure 2$)$.

\section{DISCUSSION}

There were 2 main findings to this study: (1) HFpEF patients were more at risk of mortality and need for inotropic support after cardiothoracic surgery than control patients were with preserved ejection fraction and (2) this association between $\mathrm{HFpEF}$ and increased postoperative risk remained significant when adjusting for EuroSCORE II (ie, for any given EuroSCORE II). Patients with HFpEF were at higher risk of adverse postoperative outcomes.

\section{HFpEF and Postoperative Mortality}

This study aimed to assess the prognostic impact of HFpEF on postoperative in-hospital mortality after cardiothoracic surgery. Patients HFpEF had increased postoperative mortality compared with control patients, and this finding remained true for any given EuroSCORE II. Previous publications aiming to assess the effects of HFpEF on postoperative mortality found conflicting results, although not achieving all criteria for HFpEF as defined by most recent guidelines. ${ }^{9,13}$ In a small cohort of 43 patients ( 8 of whom with HFpEF), HFpEF was not found to be associated with increased mortality in aortic valvular replacement, ${ }^{4}$ whereas it was found to be associated with poorer outcomes after CABG surgery. ${ }^{3}$ Interestingly, the latter study found similar death rates between patients with HFpEF and those with HFrEF. In the present study, although sample size did not allow for significant results, subgroup analysis of type of surgery confirmed there was a trend toward and increased risk for HFpEF patients in aortic valve surgery and mitral valve surgery. Surprisingly, in isolated $\mathrm{CABG}$ procedures, difference in mortality was less significant than for other types of procedures, whereas HFpEF had been previously identified as a risk factor of mortality in this setting. Lack of power might explain this difference; hence, further research may be necessary to investigate this issue.

\section{HFpEF and Postoperative Risk of Clinical Outcomes}

Overall, this study found that patients with HFpEF had worse outcomes compared with control patients who did not fulfill criteria for HFpEF but had LVEF $\geq 50 \%$. Interestingly, this association remained true for any given EuroSCORE II (which already accounts for the known risk factors of postoperative shock, such as age, type of surgery, preoperative state and diabetes). Several mechanisms are certainly involved, although the increased incidence of postoperative shock might suggest a few.

Diastolic dysfunction is known to be associated with increased mortality in postoperative cardiac surgery. ${ }^{12,14}$ Several factors can be implicated: inadequate myocardial protection during surgery in left ventricular hypertrophy patients ${ }^{15}$ and LV concentric hypertrophy. ${ }^{16,17}$

Variations of filling conditions in patients undergoing cardiac surgery with a diastolic dysfunction and normal LVEF might explain the higher rate of mortality in this population. $^{12}$

Right ventricular dysfunction can also be involved in the increased mortality after cardiac surgery. Indeed, some HFpEF patients might also experience right ventricular failure, which is not a condition captured by the EuroSCORE II (as only systolic pulmonary arterial pressure is included in the score). ${ }^{8}$ Right ventricular failure being a known risk factor of adverse events after cardiac surgery, patients with both $\mathrm{HFpEF}$ and right ventricular failure may be more likely to develop postoperative shock and mortality. ${ }^{18,19}$

Moreover, elevated BNP, one of the obligatory criterion for $\mathrm{HFpEF},{ }^{9}$ is associated with postoperative systemic inflammation. ${ }^{20}$ This temporary systemic inflammatory response syndrome could explain the higher need of vasoactive agents after cardiac surgery in the present cohort of patients with $\mathrm{HFpEF}^{21}$

\section{LIMITATIONS}

We acknowledge some limitations to the study. First, external validation may be an issue because of the single-center nature of the design. However, the results brought forward similar mortality rates and preoperative characteristics to those reported in previous cohorts. Bias may reside in the high-volume nature of the surgical center where the patients undergo surgery, with over 1000 cardiothoracic surgeries per year. Hence, results might be applicable only in similar volume cardiac surgery centers.

Second, although the BNP criterion we used was correct in the sense that it was elevated in regard to local laboratory values, a strict definition following ESC would require a lower threshold (ie, $35 \mathrm{ng} / \mathrm{L}$ ). It must be noted that this threshold depends on laboratories, and the upper normal laboratory value in this study was $100 \mathrm{ng} / \mathrm{L}$. Moreover, ad hoc analysis showed that no patients addressed for cardiac surgery had preoperative BNP values less than $35 \mathrm{ng} / \mathrm{L}$. This could indicate that a unique BNP threshold in the definition might not be pertinent in cardiac surgery setting, although the principle of "elevated" BNP would still be applicable.

Third, the heterogeneity of cardiac procedures that were involved make the interpretability of results less potent than 
if reduced to a single kind of procedure. Indeed, mitral valve is a known risk factor of mortality after cardiac surgery. ${ }^{22,23}$ However, analyses accounted for the different types of procedures and HFpEF still remained independently associated with prespecified outcomes.

Finally, HFpEF definition remains largely heterogeneous. It allows the inclusion of very different types of cardiac diseases, explaining why a single mechanism cannot explain the increased postoperative mortality rate by itself. This study included the broader spectrum of HFpEF, adhering to ESC guidelines definitions to account for this heterogeneity. The number of patients required to perform subgroup analyses per type of cardiac disease did not allow for such investigations. This calls for further studies to better assess specific postoperative risk.

\section{CONCLUSIONS}

HFpEF was associated with increased mortality and shock after cardiothoracic surgery. This association remained significant when adjusting for EuroSCORE II-that is, for any given EuroSCORE II, patients with $\mathrm{HFpEF}$ were at higher risk.

\section{Conflict of Interest Statement}

Authors have nothing to disclose with regard to commercial support.

\section{References}

1. Owan TE, et al. Trends in prevalence and outcome of heart failure with preserved ejection fraction. New Engl J Med. 2006;355:251-9.

2. Roques F, et al. Risk factors and outcome in European cardiac surgery: analysis of the EuroSCORE multinational database of 19030 patients. Eur J Cardiothorac Surg. 1999; 15:816-23.

3. Marui A, et al. Comparison of 5-year outcomes after coronary artery bypass grafting in heart failure patients with versus without preserved left ventricular ejection fraction (from the CREDO-Kyoto CABG Registry Cohort-2). Am J Cardiol. 2015;116:580-6.

4. Nakagawa D, et al. Postoperative outcome in aortic stenosis with diastolic heart failure compared to one with depressed systolic function. Int Heart J. 2007;48: 79-86.

5. Lam CSP, et al. Epidemiology and clinical course of heart failure with preserved ejection fraction. Eur J Heart Failure. 2011;13:18-28.

6. Shah SJ, et al. Baseline characteristics of patients in the treatment of preserved cardiac function heart failure with an aldosterone antagonist trial. Circ Heart Fail. 2013;6:184-92.

7. Tribouilloy $\mathrm{C}$, et al. Prognosis of heart failure with preserved ejection fraction: a 5-year prospective population-based study. Eur Heart J. 2008;29:339-47.
8. Nashef SA, et al. EuroSCORE II. Eur J Cardiothorac Surg. 2012;41:734-44; discussion 744-5.

9. Ponikowski P, et al. 2016 ESC guidelines for the diagnosis and treatment of acute and chronic heart failure. The task force for the diagnosis and treatment of acute and chronic heart failure of the European Society of Cardiology (ESC). Developed with the special contribution of the Heart Failure Association (HFA) of the ESC. Eur Heart J. 2016;37:2129-200.

10. Devereux RB, et al. Performance of primary and derived M-mode echocardiographic measurements for detection of left ventricular hypertrophy in necropsied subjects and in patients with systemic hypertension, mitral regurgitation and dilated cardiomyopathy. Am J Cardiol. 1986;57:1388-93.

11. Dalen M, et al. Survival after coronary artery bypass grafting in patients with preoperative heart failure and preserved vs reduced ejection fraction. JAMA Cardiol. 2016;1:530-8

12. Salem R, et al. Left ventricular end-diastolic pressure is a predictor of mortality in cardiac surgery independently of left ventricular ejection fraction. Br J Anaesth. 2006;97:292-7.

13. Oktay AA, Shah SJ. Diagnosis and management of heart failure with preserved ejection fraction: 10 key lessons. Curr Cardiol Rev. 2015;11:42-52.

14. Licker M, et al. Preoperative diastolic function predicts the onset of left ventricular dysfunction following aortic valve replacement in high-risk patients with aortic stenosis. Critical Care. 2010;14:R101.

15. Natsuaki M, et al. Risk factors associated with perioperative myocardial damage in patients with severe aortic stenosis. J Cardiovasc Surg. 2004;45 $271-7$.

16. Duncan AI, et al. Influence of concentric left ventricular remodeling on early mortality after aortic valve replacement. Ann Thorac Surg. 2008;85:2030-9.

17. Orsinelli DA, et al. Left ventricular hypertrophy and mortality after aortic valve replacement for aortic stenosis. A high-risk subgroup identified by preoperative relative wall thickness. J Am Coll Cardiol. 1993;22:1679-83.

18. Garatti A, et al. Impact of right ventricular dysfunction on the outcome of heart failure patients undergoing surgical ventricular reconstruction. Eur J Cardiothorac Surg. 2015;47:333-40; discussion 340.

19. Haddad F, et al. Right ventricular myocardial performance index predicts perioperative mortality or circulatory failure in high-risk valvular surgery. J Am Soc Echocardiogr. 2007;20:1065-72.

20. Kerbaul F, et al. High concentrations of N-BNP are related to non-infectious severe SIRS associated with cardiovascular dysfunction occurring after off-pump coronary artery surgery. Br J Anaesth. 2004;93:639-44.

21. Sablotzki A, et al. The systemic inflammatory response syndrome following cardiac surgery: different expression of proinflammatory cytokines and procalcitonin in patients with and without multiorgan dysfunctions. Perfusion. 2002;17:103-9.

22. Bakaeen FG, Shroyer AL, Zenati MA, Badhwar V, Thourani VH Gammie JS, et al. Mitral valve surgery in the US Veterans Administration health system: 10-year outcomes and trends. J Thorac Cardiovasc Surg. 2017:155:105-17.e5.

23. Schnittman SR, et al. Survival and long-term outcomes after mitral valve replacement in patients aged 18 to 50 years. J Thorac Cardiovasc Surg. 2018; 155:96-102.e11.

Key Words: heart failure with preserved ejection fraction, risk-score, perioperative management, risk evaluation, cardiac surgery 


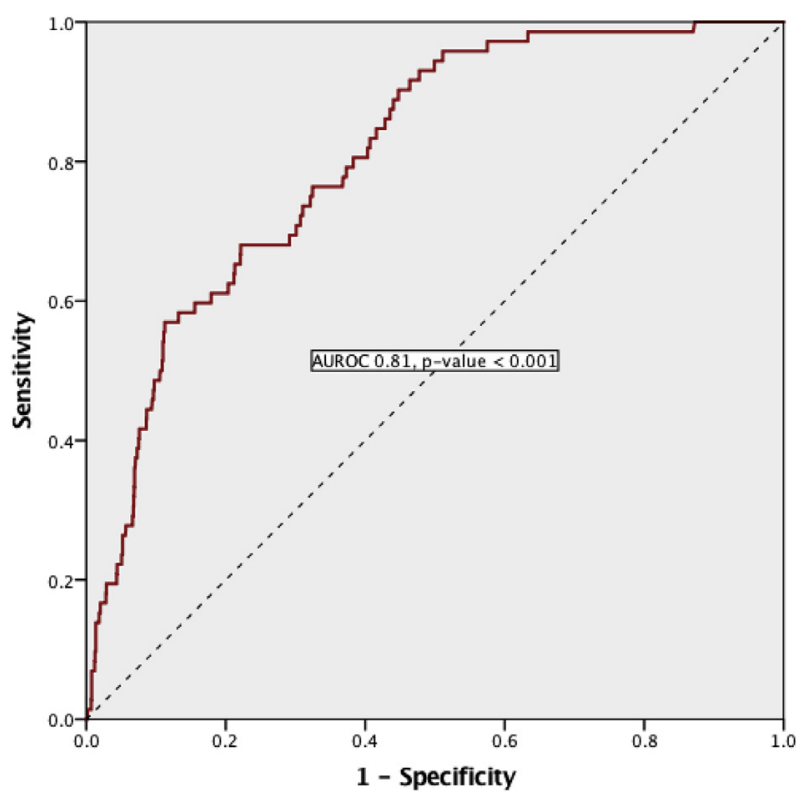

FIGURE E1. Receiver operating characteristic curve of EuroSCORE II regarding in-hospital mortality after surgery. EuroSCORE, European System for Cardiac Operative Risk; AUROC, receiver operating characteristics area under the curve.

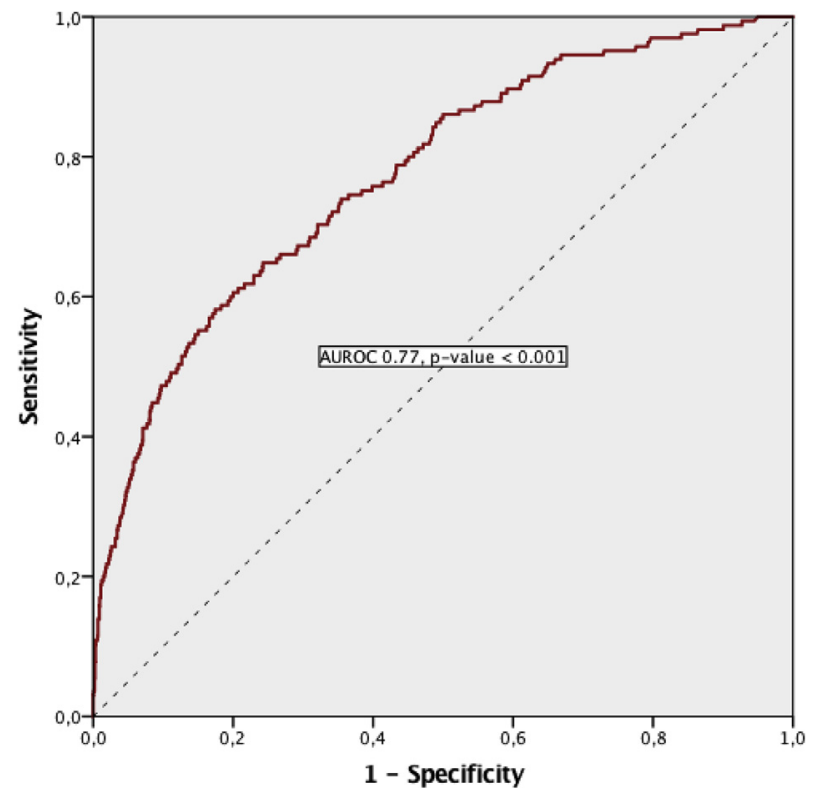

FIGURE E2. Receiver operating characteristic curve of EuroSCORE II regarding postoperative need for inotropic support. EuroSCORE, European System for Cardiac Operative Risk; AUROC, receiver operating characteristics area under the curve. 
TABLE E1. Multivariate analysis regarding in-hospital mortality after surgery

\begin{tabular}{lcccc}
\hline \multicolumn{1}{c}{ Variables } & Unadjusted. hazard ratio & Adjusted hazard ratio & $\mathbf{9 5 \%}$ confidence interval & Adjusted $\boldsymbol{P}$ value \\
\hline Age $($ per y) & 1.06 & 1.06 & $1.03-1.09$ & $1.00-2.98$ \\
HFpEF & 1.86 & 1.73 & $1.28-4.16$ & .001 \\
Mitral valve procedure & 2.26 & 2.30 & $3.72-14.54$ & .048 \\
Emergency procedure & 8.40 & 7.35 & $1.32-5.93$ & -006 \\
Thoracic aorta procedure & 2.95 & 2.80 & - & -001 \\
eGFR $<50 \mathrm{~mL} / \mathrm{min}$ & 2.56 & - & - & - \\
sPAP $>55 \mathrm{~mm} \mathrm{Hg}$ & 3.63 & - & - \\
\hline
\end{tabular}

$H F p E F$, Heart failure with preserved ejection fraction; $e G F R$, estimated glomerular filtration rate; $s P A P$, systolic pulmonary artery pressure.

TABLE E2. Multivariate analysis regarding postoperative need for inotropic support

\begin{tabular}{lcccc}
\hline \multicolumn{1}{c}{ Variables } & Unadjusted. odds ratio & Adjusted odds ratio & $\mathbf{9 5 \%}$ confidence interval & Adjusted $\boldsymbol{P}$ value \\
\hline $\mathrm{HFpEF}$ & 2.99 & 1.52 & $1.04-2.23$ & .029 \\
Emergency procedure & 3.52 & 3.77 & $2.05-6.95$ & $<.54-3.13$ \\
eGFR $<50 \mathrm{~mL} / \mathrm{min}$ & 3.04 & 2.20 & $2.87-7.76$ & $<.001$ \\
sPAP $>55 \mathrm{~mm} \mathrm{Hg}$ & 9.27 & 4.72 & $0.26-0.63$ & - \\
Isolated CABG & 0.24 & 0.41 & - & $<.001$ \\
Age (per y) & 1.03 & - & - & - \\
$\geq 3$ combined procedures & 3.81 & - & - & - \\
\hline BNP & 1.006 & - & - \\
\hline
\end{tabular}

$H F P E F$, Heart failure with preserved ejection fraction; $C A B G$, coronary artery bypass graft; $B N P$, brain natriuretic peptide. 